\title{
Slow dynamics of a confined supercooled binary mixture: direct space analysis
}

\author{
P. Gallo ${ }^{\dagger *}$, R. Pellarin ${ }^{\dagger}$ and M. Rovere ${ }^{\dagger}$, \\ $\dagger$ Dipartimento di Fisica, Università "Roma Tre", \\ Istituto Nazionale per la Fisica della Materia, \\ Unità di Ricerca Roma Tre \\ Via della Vasca Navale 84, 00146 Roma, Italy.
}

\begin{abstract}
Dynamical properties of a Lennard-Jones binary mixture embedded in an off lattice matrix of soft spheres are studied in the direct space upon supercooling by molecular dynamics simulations. On lowering temperature the smaller particles tend to avoid the soft sphere interfaces and correspondingly their mobility decreases below the one of the larger particles. The system displays a dynamic behaviour consistent with the Mode Coupling predictions. A decrease of the mode coupling crossover temperature with respect to the bulk is found. We find however that the range of validity of the theory shrinks with respect to the bulk. This is due to the change in the smaller particle mobility and to a substantial enhancement of hopping processes well above the cross over temperature upon confinement.
\end{abstract}

PACS numbers: 61.20.Ja, 61.20.Lc, 64.70.Pf

\section{INTRODUCTION}

Liquids under confinement represent a field of growing interest in science due to the connection with relevant technological and biophysical problems ${ }^{1}$. In this field modifications of both the phase diagram and dynamics of the confined liquid with respect to the bulk represent a key point, in particular as far as the possibility of supercooling is concerned 2 . Experiments exhibit a very diversified phenomenology for supercooled confined liquids. In particular the calorimetric glass transition temperature, $T_{g}$, can increase $\mathrm{e}^{\frac{3}{}}$, decrease $\mathrm{e}^{4.5}$ or be unaffected ${ }^{6}$ with respect to the the bulk value depending on the liquid, the confining geometry and the nature of the substrate.

Phenomenological arguments predict the existence of domains of cooperative dynamics in the supercooled liquid $^{7}$. The size of these domains is expected to grow upon supercooling. The existence of an upper bound for the domain size in confined media implies a decrease of $T_{g}$ upon confinement. In fact if this size remains small on supercooling then the probability of a cooperative motion, and therefore of a structural relaxation, is large, and dynamics is faster.

The mode coupling theory of the glass transition $(\mathrm{MCT})^{8}$ is able to describe the dynamics of bulk liquids in the supercooled region on approaching a crossover temperature $T_{C}$. At $T_{C}$ the system passes from a regime where ergodicity is attained through structural relaxations to a regime where this mechanism is frozen and only activated processes permit the exploration of the configurational space. $T_{C}$ can be estimated through experiments and computer simulations or predicted by the theory.

\footnotetext{
*Author to whom correspondence should be addressed; e-mail: gallop@fis.uniroma3.it
}

Above $T_{C}$ the relaxation mechanism of the supercooled liquid can be described as mastered by the cage effect. Nearest neighbors surround and trap the tagged particle forming a cage around it. When the cage relaxes, due to cooperative motions, the particle moves. MCT translates this phenomenological description into a precise mathematical framework where, starting from the classical equation of motion for the density correlator $\phi_{q}(t)$, a retarded memory function is introduced. In the idealized version of MCT hopping processes are neglected and the retarded non linear set of integro-differential equations can be solved analytically to the leading order in $\epsilon=\left(T-T_{C}\right) / T_{C}$, deriving universal results for the density correlator. With these approximations $T_{C}$ is the temperature of structural arrest of the ideal system. The success of this theory is due to the fact that on approaching $T_{C}$ from above hopping processes can be neglected in many liquids and the predictions of the idealized version of MCT hold.

Molecular dynamics studies of model liquids in restricted geometries intended to assess the applicability of $\mathrm{MCT}$ can give an important contribution to the characterization of the glass transition scenario in confinement. In this field recent progresses have been done for water confined in a silica nano pore ${ }^{9.10}$ and for confined thin polymer films 11 .

We performed a Molecular Dynamic (MD) study of the single particle dynamical properties of a LennardJones binary mixture (LJBM) embedded in an off lattice matrix of soft spheres. The model considered represents a situation of strong confinement analogous to real silica xerogels $12,13,14$. The bulk phase of the chosen mixture is known to test most of the MCT predicted features 15 . A brief report on some of our findings has been recently published 16 .

We present in this paper direct space quantities evaluated from MD trajectories in order to define the range of validity of MCT with respect to the bulk phase. In the next section we describe the model adopted and the 
details of the MD simulations. In the third section we illustrate the radial pair distribution functions and the coordination numbers of the system. The fourth section is devoted to the study of the dynamic quantities in the direct space: the mean square displacement (MSD), the van Hove self correlation function and the non-Gaussian parameter. Last section contains the concluding remarks.

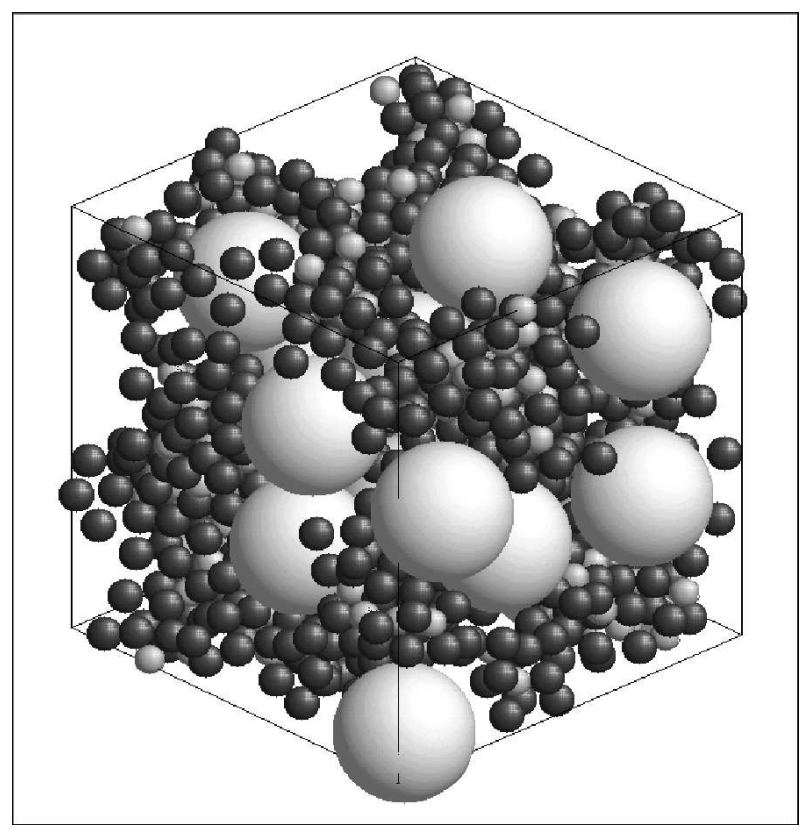

FIG. 1: Graphic representation of the model studied. Small light gray particles are of A type, small dark gray particles are of B type. The soft spheres that form the confining system appear in the figure as large dark gray spheres. The cube in which the system is embedded is the simulation box. The radii of the three types of particles are purely representative. The $\sigma$ values are listed in Tab凹

\section{MODEL AND MOLECULAR DYNAMICS}

The confining medium consists in a rigid disordered array of soft spheres. The simulation box contains 16 soft spheres labeled in the following with the letter M. The interspaces of this structure host a liquid LJBM of 1000 particles, composed of 800 particles of type A and 200 particles of type B. The interaction pair potential can be written in the following general form:

$$
V_{\mu \nu}(r)=4 \epsilon_{\mu \nu}\left[\left(\frac{\sigma_{\mu \nu}}{r}\right)^{12}-\eta_{\mu \nu}\left(\frac{\sigma_{\mu \nu}}{r}\right)^{6}\right]
$$

where indexes $\mu, \nu$ run on the particle types A,B,M. The parameters for the three components are listed in Table I The parameters of the LJBM have been chosen as in ref ${ }^{15}$. The shifted potential technique has been used with a cutoff of $2.5 \sigma_{\mu \nu}$. Periodic boundary conditions are applied. In the following Lennard-Jones units will be used, therefore energy will be given in units of

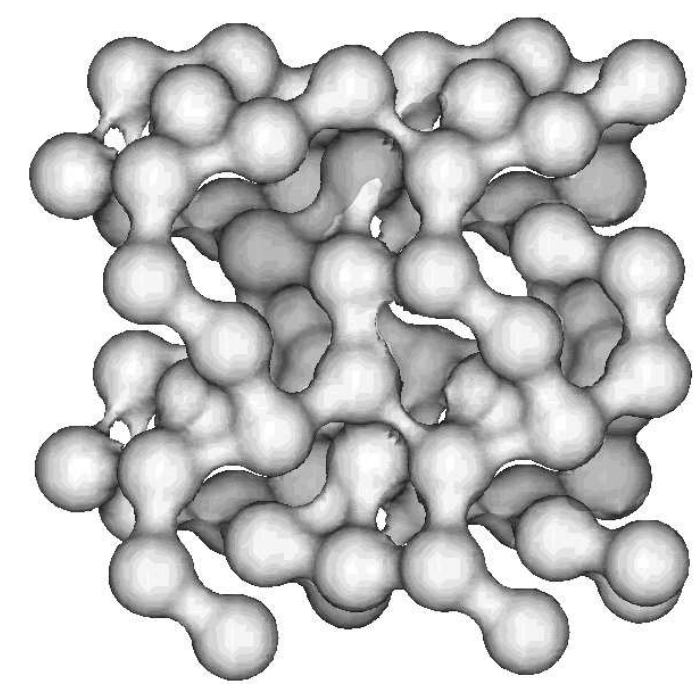

FIG. 2: Graphic representation of the confining disordered structure. The sixteen spheres of the matrix contained in the simulation box of Fig 1 have been repeated to obtain a box of height $2 \mathrm{~L}$, width $2 L$ and depth $L$. The surface that appears in the figure is equipotential.

$\epsilon_{A A}$, temperature in units of $\epsilon_{A A} / K_{B}$, length in units $\sigma_{A A}$ and time in units of $\left(m \sigma_{A A}^{2} /\left(48 \epsilon_{A A}\right)\right)^{1 / 2}$. The box length is $L=12.6$. The simulation box of the system is represented in Fig. 10 As it can be seen from the picture the LJBM is in a strongly confined environment as only few layers of particles can accommodate among the soft spheres. Fig. 2 shows a cell made of four simulation boxes. Only equipotential surfaces of the soft spheres are displayed in order to offer a clearer image of the disordered porous structure hosting the liquid and to highlight the analogies with silica xerogels.

We have conducted MD simulations of this system in the NVE ensemble. The equations of motion were solved by the velocity Verlet algorithm. The system was equilibrated at different reduced temperatures via a velocity rescaling procedure. The temperatures investigated are $T=5.0,2.0,0.8,0.58,0.538,0.48,0.465,0.43,0.41,0.39$. The timestep used for $T \geq 1$ was 0.01 and for $T<1$ was 0.02 . For the lowest temperature investigated, $T=0.37$, a production run of $14 \times 10^{6}$ timesteps was performed. We verified that the results obtained do not depend on the specific choice of the disordered matrix by running MD simulations for two other different configurations of the disordered matrix of soft spheres. Both thermodynamics and dynamics appeared the same for the three systems, in particular deviations among the diffusion coefficients extracted at given temperatures from the MSD of different configurations are within $2 \%$.

Values of thermodynamics quantities of the equilibrated systems are shown in Fig [3. Averaged pressure, potential energy and total energy per particle of the mixture behave similar to the bulk. A stability of the system throughout all the isochore explored is evident from the smoothness of the curves. 
Due to the soft spheres potential the free volume accessible for the mixture strongly depends on temperature. In Fig. 4 we show an estimate of the free volume for $\mathrm{A}$ and $\mathrm{B}$ particles in our confined system as a function of temperature. The estimate has been carried out through the Voronoi tessellation. This procedure has been applied only to non-interfacial particles. The free volume has been calculated by averaging over 160 configurations of the system for each temperature.

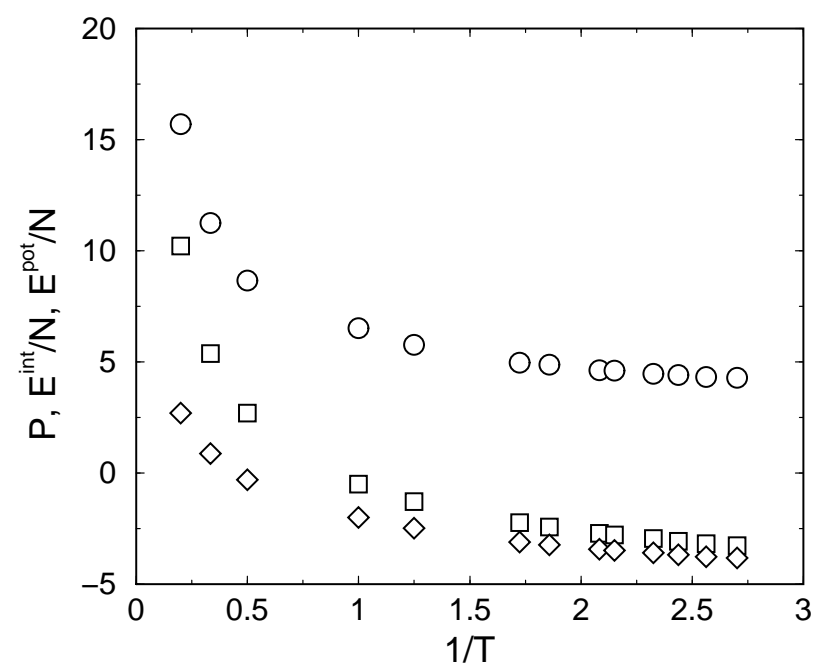

FIG. 3: Thermodynamics of the confined Lennard-Jones binary mixture: pressure (circles), total energy per particle (squares) and potential energy per particle (diamonds) versus the inverse of the temperature.

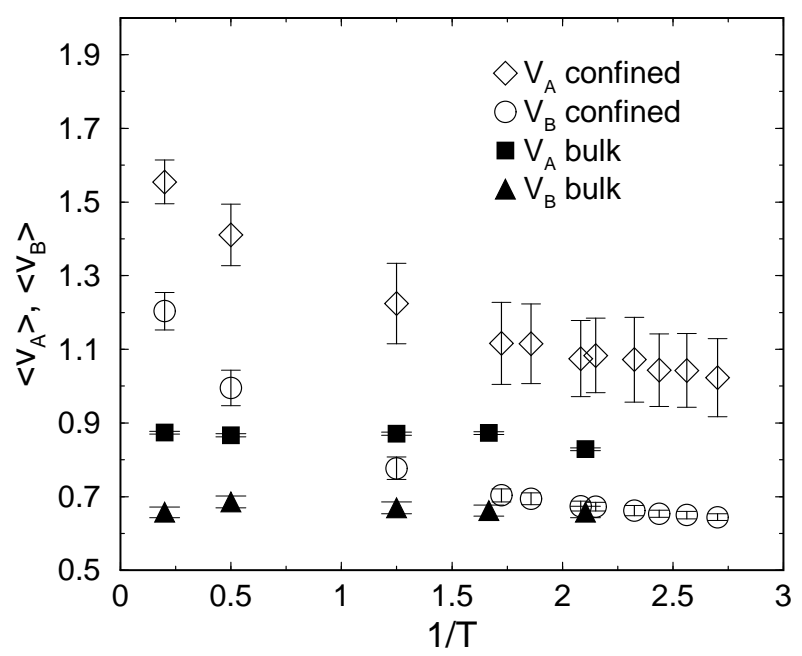

FIG. 4: Specific volumes of the Voronoi cells for A and B particles. Particles in contact with the soft confining spheres have been excluded. The bulk considered is that of ref. ${ }^{15}$.

\section{RADIAL PAIR CORRELATION FUNCTIONS}

The radial pair correlation functions $g_{\mu \nu}(r)$ are shown in Fig [5. For all the temperatures investigated the system is in the liquid phase as no abrupt sharpening of the peaks is evident. No indications of phase separation appear upon supercooling either. The latter phenomenon would in fact result in a substantial decrease of the area of the first peak, $a b_{1}$, of the $g_{A B}(r)$, which we do not observe in our system. On lowering temperature the peaks sharpen and enhance more than in the bulk due to the strong packing induced by the soft spheres. A clear element of distinction with respect to the bulk is the enhancement of intensity of the first $g_{B B}(r)$ peak, $b b_{1}$, as supercooling progresses. The same peak appeared instead reduced to a small shoulder for supercooled temperatures in the bulk.

The functions $g_{M A}(r)$ and $g_{M B}(r)$ are characteristic of this confined system and are representative of the interaction between the confining soft spheres and the particles of the mixture. We note the gradual disappearance of the first peak, $m b_{1}$, of the $g_{M B}(r)$ that begins at $T=2.0$ while the second peak, $m b_{2}$, enlarges its area. Correspondingly the first peak of the $g_{M A}(r)$ shifts to larger $r$. For lower temperatures, namely $T<0.8$, the structure of the mixture stabilizes so that MA and MB peaks alternate in space.

In order to quantify the above considerations for the radial distribution functions we calculated the coordination number defined as:

$$
N_{\mu \nu}\left(r_{1}, r_{2}\right)=4 \pi \rho x_{\nu} \int_{r_{1}}^{r_{2}} r^{2} g_{\mu \nu}(r) d r
$$

where $\rho$ is the total number density $N / V$, where $N=$ 1016, $V=L^{3}$ and $x_{\nu}$ is the relative fraction of the $\nu$ species. The quantity of Eq. 2 evaluated for each peak of the $g_{\mu \nu}(r)$, is shown in Fig. 6] The number of B particles around the soft sphere in the first shell lowers upon decreasing temperature in favor of an enhancement in the second shell. Correspondingly the number of A particles in the first shell enhances.

The above considerations on the radial distribution functions and the coordination numbers lead to the conclusion that B particles avoid the interface with the soft spheres for low temperatures. In Fig. 7 a snapshot of the system for $T=0.37$ evidences this behavior. The fluctuations of the error bars in Fig 4 also confirm this phenomenon. In fact since interfacial particles have been excluded from the calculation of the free volume a larger fluctuation corresponds to a frequent exchange of the particles with the interface.

\section{DYNAMIC QUANTITIES}

In the following we shall describe the temperature variations of the time dependent direct space quantities analyzed for our confined system upon supercooling, namely 


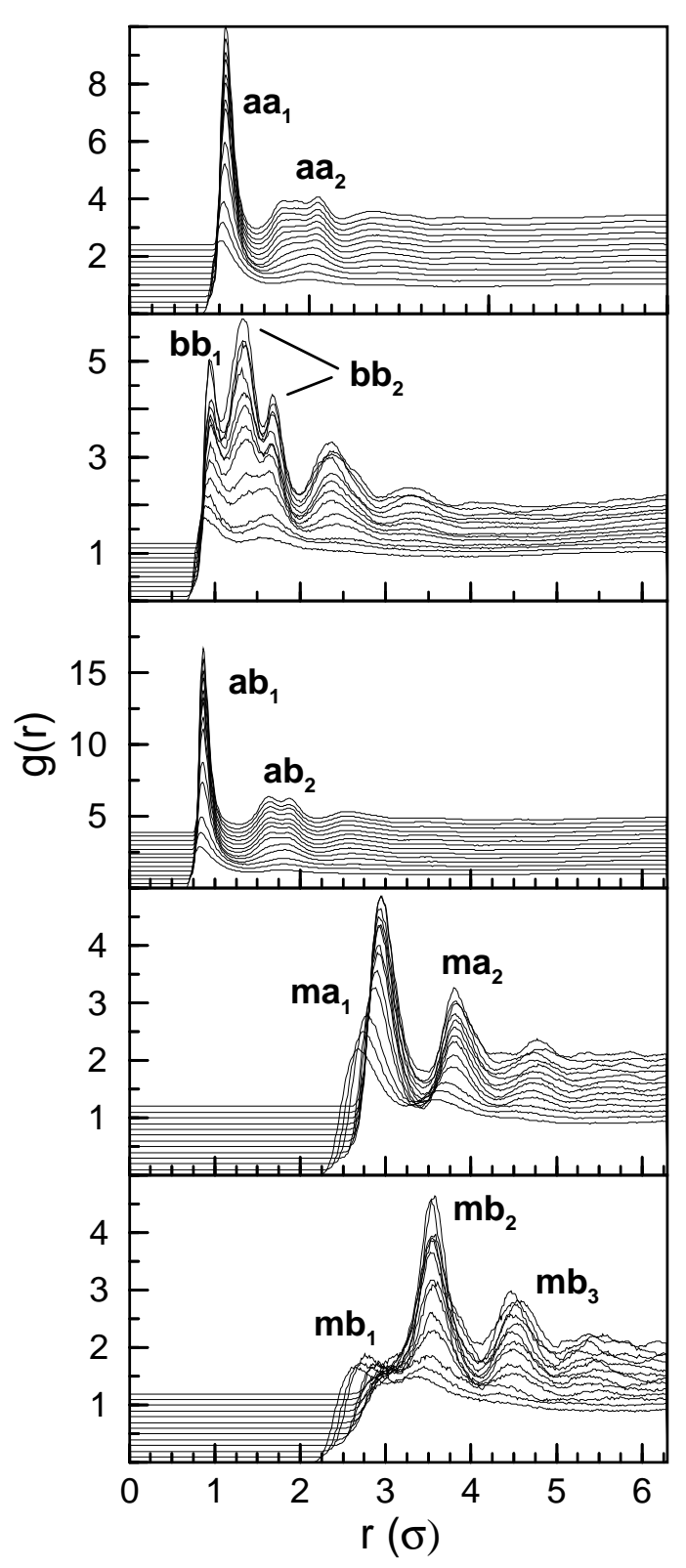

FIG. 5: Radial distribution functions calculated at different temperatures for the system. From top to bottom AA, $\mathrm{BB}, \mathrm{AB}, \mathrm{MA}$ and $\mathrm{MB}$. Lower temperatures are progressively shifted upward. For the AA function the shift is 0.2 , for $\mathrm{AB}$ 0.3 while for the remaining $\mathrm{BB}, \mathrm{MA}$ and $\mathrm{MB}$ it is 0.1 . The labeling of the peaks is used in the text and in Fig 6

the MSD, the van Hove self correlation function and the non-Gaussian parameter.

The asymptotic behaviour of the MSD can be used as a test of an MCT prediction. The MSD is in fact expected, as supercooling progresses, to develop a plateau at intermediate time scale, corresponding to the rattling of the particle in the cage. After the initial short time ballistic motion the particle enters the plateau whose extension in time enhances upon lowering temperature. After leaving the plateau region the Brownian behaviour predicted

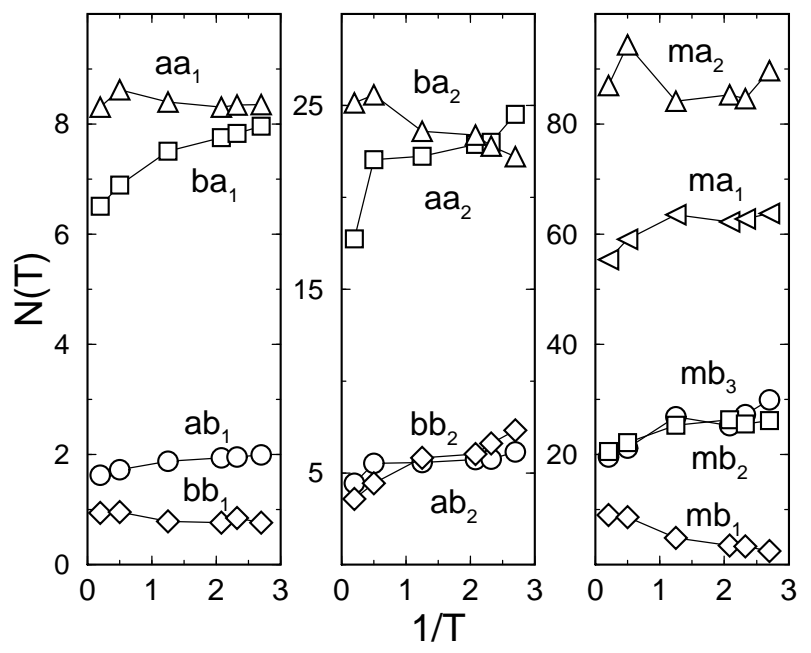

FIG. 6: Coordination numbers of the shells defined by the peaks of the radial distribution functions as labeled in Fig 5 See Eq. 2

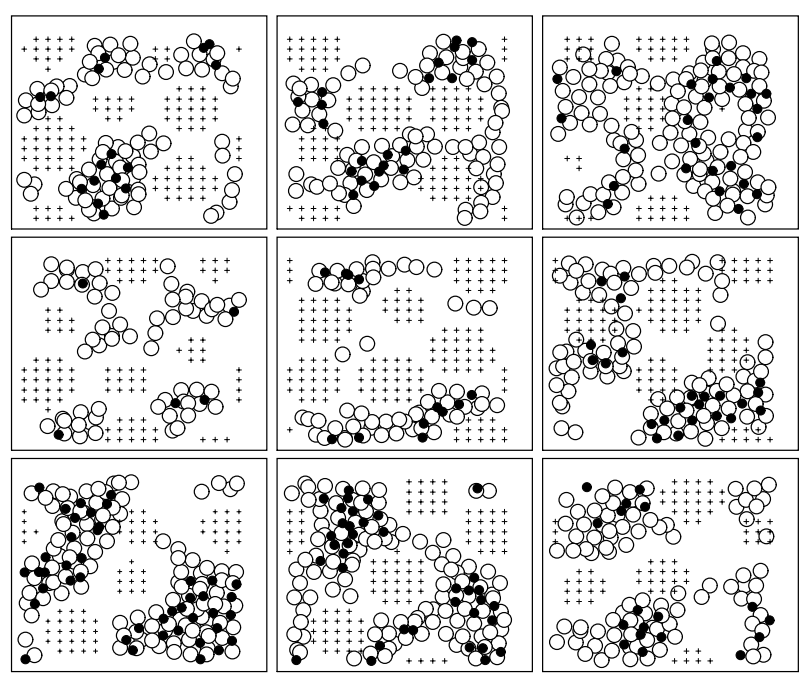

FIG. 7: Tomographic snapshots of the system at the lowest temperature investigated, $\mathrm{T}=0.37$. In order to obtain this picture the system has been divided in slices of height $1.4 \sigma$. From top to bottom and from left to right every picture displays the content of each slice. Empty circles are A-type particles, filled circles are B-type. Crosses mark the volume of the soft spheres. No phase separation is evident. At this temperature $\mathrm{B}$ particles avoid the interfacial region.

for a simple liquid in a stable state is restored. From the slope of the late MSD the diffusion constant $D$ can be extracted through Einstein relation $<r^{2}(t)>=6 D t$. The temperature behaviour of diffusion coefficients can give an estimate of the mode coupling crossover temperature through the MCT power law behaviour

$$
D \sim\left(T-T_{C}\right)^{\gamma}
$$

In Fig 8 we show the MSD calculated for both A and B particles as a function of time for all the temperatures 


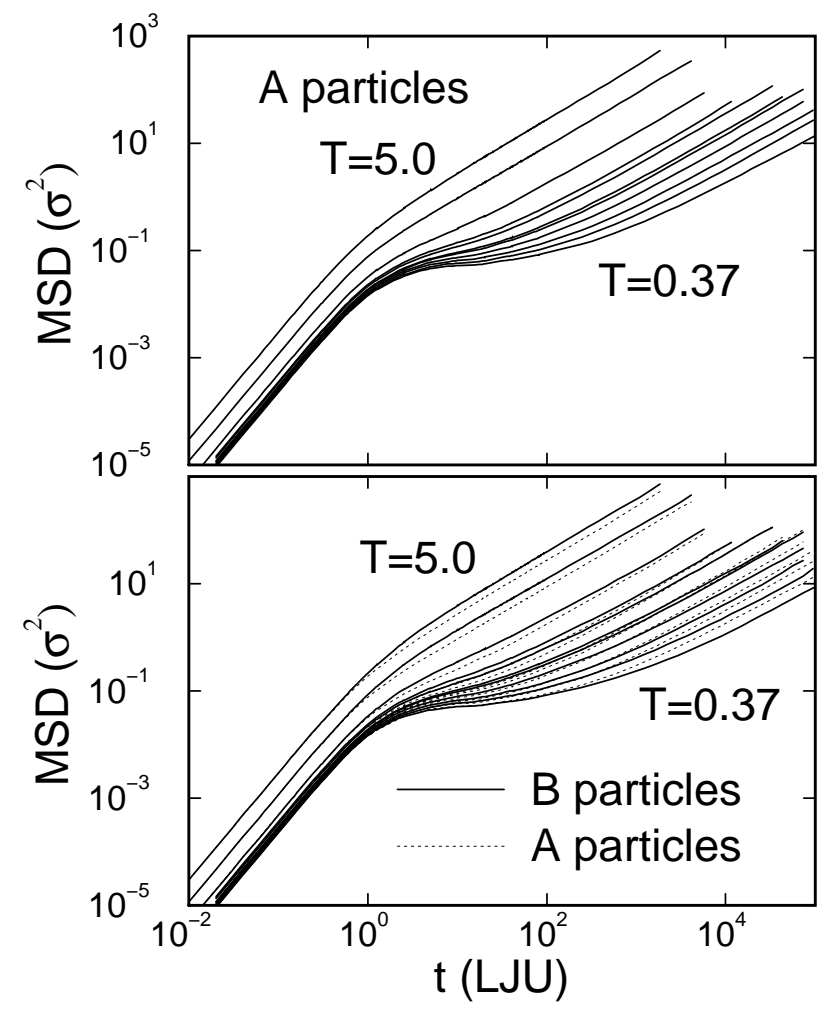

FIG. 8: Log-log plot of the mean square displacements of A particles and B particles for all the temperatures investigated, namely $T=$ $5.0,2.0,0.8,0.58,0.538,0.48,0.465,0.43,0.41,0.39$.

investigated. It can be seen that also in this confined system the binary mixture displays the typical features of a glass former. From the height of the plateau the cage radius can be extracted and the value obtained for both $\mathrm{A}$ and $\mathrm{B}$ particles is $r_{c}^{2}=0.04$, analogous to the bulk result $\frac{15}{5}$.

The lowest temperature reached in our study is $T=$ 0.370 against $T=0.466$ for the bulk. The elongation of the plateau of the MSD for our lowest temperature is comparable to that of the lowest temperature of the bulk. The diffusion coefficients extracted from the slopes are reported in Fig. 9 At higher temperatures $T>1.0$ the larger A particles diffuse slower than B particles while they move faster at lower temperatures. As shown in the previous section, in the confined case for lower temperatures B particles tend to avoid the interface of the soft spheres. As a consequence they remain confined in the inner part of the channels, surrounded by A particles, and this diminishes the accessible free volume lowering mobility below the one of A particles, see also Fig प

In Fig. 9 the fit to the MCT power law of Eq. 3 is also reported. The estimate of the crossover temperature given by the fit is $T_{C}=0.356$ for both $\mathrm{A}$ and $\mathrm{B}$ particles. The exponents are $\gamma=1.86$ and 1.89 respectively for A and B particles. We do observe deviation from a power law both at high and low tempera-

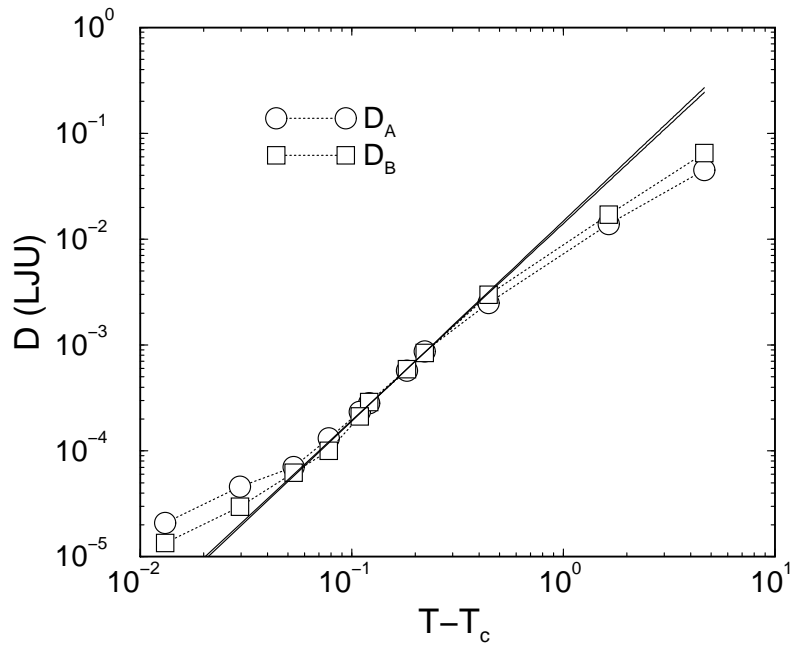

FIG. 9: Diffusion coefficients for A and B particles (symbols). Continuous lines correspond to the fit to Eq. 3. The values of the parameters extracted from the fit are $T_{C}=0.356$ for both species and $\gamma=1.86$ for A particles and $\gamma=1.89$ for $\mathrm{B}$ particles.

tures. The temperatures used for the fit are in the range $0.410 \leq T \leq 0.58$ corresponding to $0.153<\epsilon<0.631$. The range of validity of MCT reported for the bulk is much wider, $0.07<\epsilon<1.30$. The upper limit is generally due in bulk to the non-validity of the asymptotic expansion for too large values of $\epsilon$. Here we infer that a further decrease of this limit is due to the progressive B particles avoidance of the soft sphere interfaces upon supercooling. For the temperatures below the upper limit the peaks of $\mathrm{g}(\mathrm{r})$ for MA and MB have in fact already stabilized, see Fig 5 The deviation observed at the two lowest temperatures is to be connected to the presence of hopping mechanisms, analogously to bulk liquids, as we will see in more detail when we illustrate the behaviour of the VHSCF.

The Van Hove self correlation function, VHSCF, is defined, for a system of $\mathrm{N}$ particles, as

$$
G_{S}(r, t)=\frac{1}{N}\left\langle\sum_{i=1}^{N} \delta\left[\mathbf{r}+\mathbf{r}_{i}(0)-\mathbf{r}_{i}(t)\right]\right\rangle
$$

$4 \pi r^{2} G_{S}(r, t) d r$ is the probability of finding a particle at distance $r$ after a time $t$ if the same particle was at the origin $r=0$ at the initial time $t=0$.

In Fig 10 we show $4 \pi r^{2} G_{S}(r, t) d r$ at fixed times for three different temperatures. On this scale the correlators of A and B particles do not differ substantially. At high temperature the VHSCF decays regularly as time increases as expected for a simple liquid. As supercooling progresses the system begins to display a new feature that is best evident for the lowest temperature. This feature consists in the clustering of the curves at intermediate times which is the signature of the caging of the particles. The zone where the peak value clusters is 
$r=0.15-0.25$ and corresponds to the zone in which the MSD flattens. For the lowest temperature investigated the maximum and minimum times for the clustering are $2.6 \leq t \leq 82.0$. These values define the so called $\beta$ relaxation temporal region. In the comparison between the VHSCF of A and B particles it can be observed the crossing between the velocity of the two species. At high temperatures, in fact, the VHSCF of B particles extends further than the one of A particles for long times, while for low temperatures behaviors are swapped.

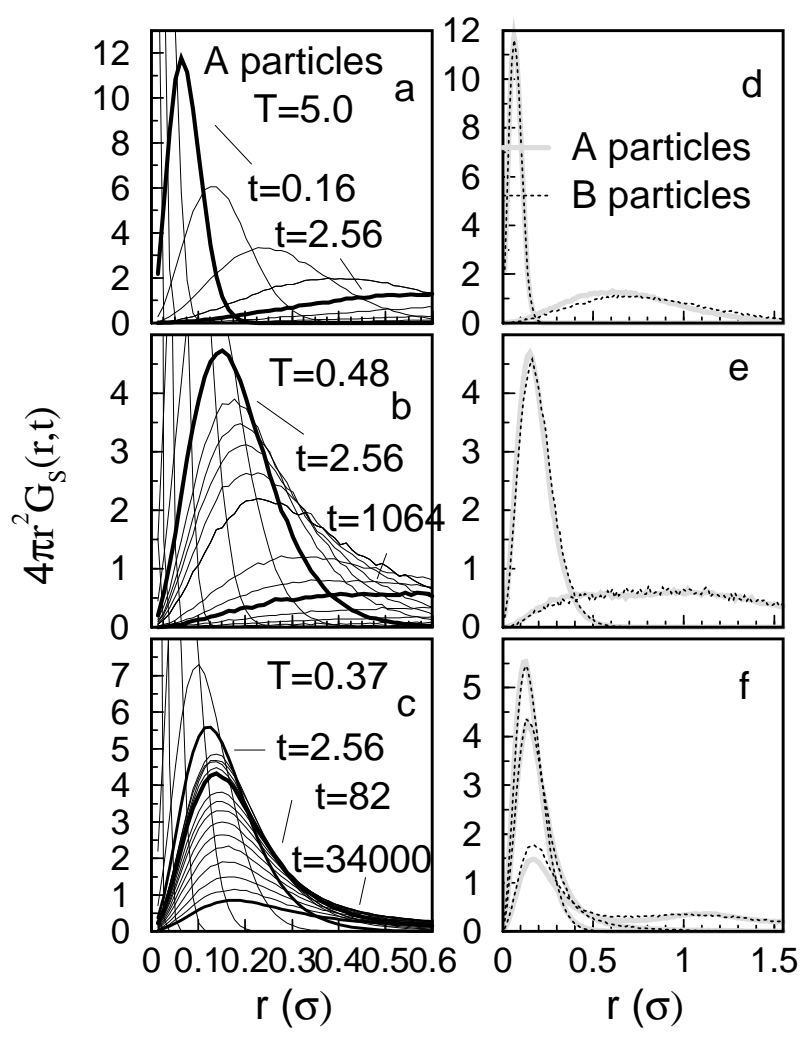

FIG. 10: $4 \pi r^{2} G_{S}(r, t) d r$ vs r. a) b) and c) pictures refer to particle $\mathrm{A}$ for $\mathrm{T}=5.0,0.5$ and 0.48 respectively. Data are sampled at fixed times. The times chosen follow the progression $t=2^{n}$. In the pictures d) e) and f) the correlators are shown for B particles (dashed lines) together with the correlators for A type for a comparison (continuous line). The curves chosen for a comparison are those indicated with a thicker line in the graphs on the left.

A behavior not present in the bulk can be observed for the lower temperatures by looking at the peak of the VHSCF for times longer than the plateau region. In this temporal range the peak of the VHSCF is expected, in the framework of MCT, to shift to larger $r$ for large times. The absence of this shift in our confined mixture, as we shall see in the next picture, must be connected with an important hopping phenomenon able to restore diffusive motions when the cage is not jet dissolved.

In Fig[11 we show a blow up of the tails of the curves of Fig 10 Aside the previously cited cage peak, we note the presence of a hopping peak around $r=1.0$. This

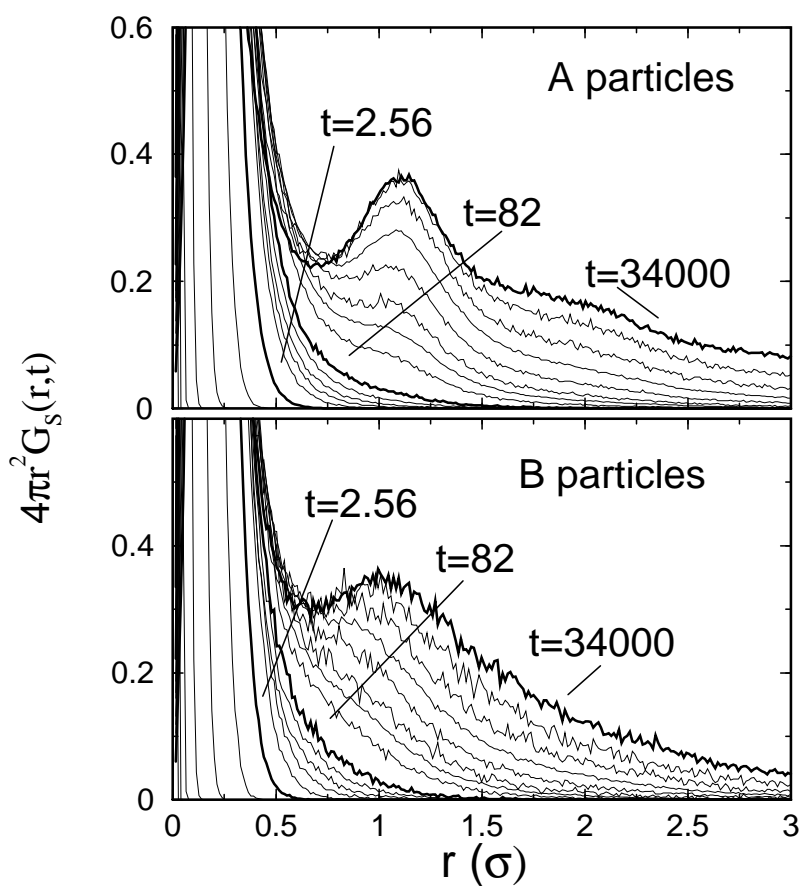

FIG. 11: Blow up of the $4 \pi r^{2} G_{S}(r, t) d r$ vs $\mathrm{r}$ for $\mathrm{T}=0.37$ for different times for A (top graph) and B (bottom graph) particles. Evidence is put on the region in which the peaks related to the presence of hopping appear.

peak enhances for late time and it is more pronounced for A particles than for B. In the bulk on approaching $T_{C}$ from above a hopping peak appears only for B particles. From the MSD it is evident that our hopping phenomena can not be neglected at the two lowest temperatures, namely at $T=0.37,0.39$. As a consequence these temperatures can not be accounted for a test of the MCT in the region around the cage relaxation time, $\alpha$ relaxation region. Nevertheless these temperatures can be used to test the theory in the $\beta$-time region. In fact, see also ref ${ }^{15}$, hopping peaks do not affect curves in the time range of the clustering region.

For the A particle a second broader peak is visible in Fig [11] This peak is also connected to the presence of hopping. The two hopping peaks correspond to the first and second peak of the $g_{A A}(r)$, see Fig [6] meaning that there are some privileged position for the atoms to be reached and that those atoms that reached these positions have traveled more than the average. Similar, the hopping peak of the B VHSCF corresponds to the first peak of the $g_{B B}(r)$. Hopping phenomena are connected with the existence of dynamical heterogeneities in supercooled liquids 17 . In confinement they appear to have above $T_{C}$ a more important role than in the bulk.

Dynamical heterogeneities are also known to cause a non gaussianity of atomic displacements. We evaluated the first non-Gaussian parameter (NGP), that works as a quantifier of the degree of non-Gaussianity. This pa- 


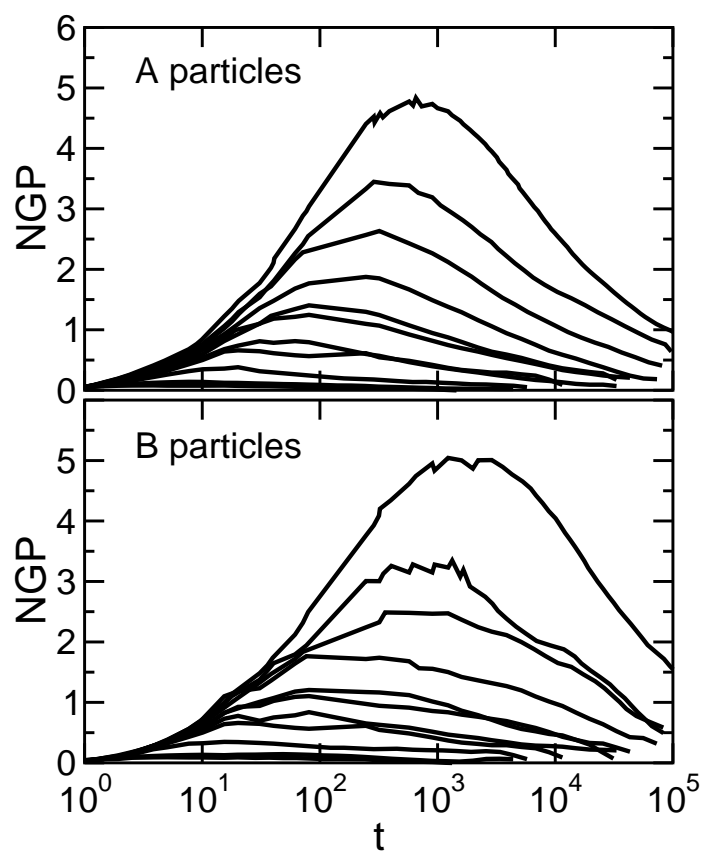

FIG. 12: Non Gaussian parameter calculated for A (top) and B (bottom) particles for all the temperatures investigated. In both graphs curves on the top correspond to lower temperatures.

rameter is defined ${ }^{18}$ in three dimension as

$$
\alpha_{2}(t)=\frac{3<r^{4}(t)>}{5<r^{2}(t)>^{2}}-1
$$

This quantity calculated for our system is reported in fig 12. Also for this parameter we have marked differences with respect to the bulk that reflect the different weight of the hopping processes in the two cases. In the bulk the NGP of $\mathrm{A}$ and $\mathrm{B}$ particles were different and never exceeded the values of 2 for A particles (no hopping above $T_{C}$ ) and 3.2 for $\mathrm{B}$ particles. Here instead we have similar NGP for A and B particles and the value grows enormously as we supercool. For the last temperature investigated the peak is around 5. We found no relation between the peak positions and MCT power law of Eq 3 as it was found for example in bulk water ${ }^{19}$ and the curves of the confined NGP do not appear to follow a master curve before the maximum as found in bulk liquids 15.19 . The trend of the NGP of bulk and confined mixture depicts a scenario in which MCT behaviour causes a mild deviation from gaussianity. The peak is around 2 when close to $T_{C}{ }^{15.19}$, its position shifts substantially upon supercooling and the NGP follows a master curve in the temporal region before the peaks. Hopping appears to cause a marked deviation from gaussianity instead, leading to a disappearance both of the MCT master curve and of the shift of the peaks upon supercooling.

\section{SUMMARY AND CONCLUSIONS}

We presented an MD study of a LJBM confined in a disordered array of soft spheres. The direct space quantities have been analyzed upon supercooling for a test of the MCT behaviour.

Two main differences with respect to the bulk mixture appear due to confinement. The $g(r)$ have evidenced that smaller B particles tend to avoid interfaces at low $\mathrm{T}$ and correspondingly their diffusion coefficient becomes lower than that of the A particles. Hopping processes are present also for $\mathrm{A}$ particle above $T_{C}$ as shown in the VHSCF.

Not obviously, these differences do not prevent the MCT asymptotic predictions from holding for this mixture also in this kind of confinement. The range of validity of MCT in the region of the $\alpha$ relaxation is however much more limited. The range of the bulk 15 is $0.07<\epsilon<1.30$ against the $0.153<\epsilon<0.631$ found in our confined model. Disturbances in the dynamical behaviour of the bulk introduced by a different type of confinement are also reported to lead to a complete disappearance of $\mathrm{MCT}^{21}$.

Power law fit to the diffusion coefficient extracted from the slope of the MSD give an estimate of $T_{C}=0.356$ for both species and $\gamma=1.86$ for A particles and $\gamma=1.89$ for B particles in agreement with MCT that states that these quantities should be the same for both A and B particles. The values of the bulk extracted from $\mathrm{D}$ in literature $\operatorname{are}^{15} T_{C}=0.435$ for both species and $\gamma=2.0$ for A particles and $\gamma=1.7$ for B particles. The exponents $\gamma$ appear similar while we have a reduction of $T_{C}$ upon confinement. A complete test of the correlators in the $\mathrm{Q}$ space will be presented in a subsequent paper ${ }^{20}$.

Acceleration of dynamics in presence of repulsive confinement is expected ${ }^{11}$ based on the fact that in confinement the cooperative rearranging region cannot grow beyond the size of the system. In the case of an attractive interaction this effect might superpose to the often severe slowing down caused by the substrate to the closest layers of the liquid ${ }^{9.10}$.

In the present work we have considered a much more complex system than the bulk. Therefore the fact the MCT could be used in this context as an unifying theoretical approach is highly relevant as a guideline for the systematic study of the important phenomenology of confined and interfacial liquids.

\section{ACKNOWLEDGMENTS}

P.G. wishes to thank J. Baschnagel,W. Götze and F. Varnik for very useful discussions. 
TABLE I: Parameters of the Lennard-Jones and soft spheres potentials as defined in Eq1 In the Table $A$ and $B$ refer to the particle of the binary mixture while $M$ refers to the confining soft spheres. Values are expressed in Lennard-Jones units.

\begin{tabular}{cccc} 
& $\sigma$ & $\epsilon$ & $\eta$ \\
\hline$A A$ & 1.0 & 1.0 & 1 \\
$B B$ & 0.88 & 0.5 & 1 \\
$A B$ & 0.8 & 1.5 & 1 \\
$M A$ & 3.0 & 0.32 & 0 \\
$M B$ & 2.94 & 0.22 & 0
\end{tabular}

1 See for example the Proceedings of the International Workshop on "Dynamics in Confinement", Eds. B. Frick, R. Zorn, H. Büttner, J. Phys. IV 10 (2000).

2 For a review on metastable liquids see: P.G. Debenedetti, Metastable Liquids: Concepts and Principles. Princeton University Press, Princeton (1997).

3 Yu. B. Mel'nichenko, J. Schüller, R. Richert and B. Ewen, C. K. Loong, J. Chem. Phys. 103, 2016 (1995).

4 P. Pissis, A. Kyritsis, D. Daoukaki, G. Barut, R. Pelster, G. Nimtz, J. Phys. Condens. Mat. 10, 6205 (1998).

5 M. Arndt, R. Stannarius, H. Groothues, E. Hempel and F. Kremer, Phys. Rev. Lett. 79, 2077 (1997).

6 R. Richert, Phys. Rev. B 54, 15762 (1996).

7 G. Adam and J. Gibbs, J. Chem. Phys. 43, 139 (1965).

8 W. Götze and L. Sjögren, Rep. Prog. Phys. 55, 241 (1992); W. Götze in Liquids, Freezing and Glass Transition Eds. J. P. Hansen, D. Levesque, J. Zinn-Justin, North Holland, Amsterdam (1991);W. Götze, J. Phys.: Condens. Matter 11, A1 (1999).

9 P. Gallo, M. Rovere, E. Spohr, Phys. Rev. Lett. 85, 4317 (2000).

10 P. Gallo, M. Rovere, E. Spohr, J. Chem. Phys. 113, 11324

11 F. Varnik, J. Baschnagel and K. Binder, Phys. Rev. E 65, 021507 (2002).

12 M.L. Rosinberg in New Approaches to problems in liquid state theory. Eds. C. Caccamo, J.P. Hansen and G. Stell, Kluwer Academic Publ. (1999).

13 L. Sartisov and P.A. Monson Phys. Rev. E 61, 7231 (2000).

14 K.S. Page and P.A. Monson Phys. Rev. E 54, R29 (1996).

15 W. Kob and H.C. Andersen, Phys. Rev. Lett. 73, 1376(1994); Phys. Rev. E 51, 4626 (1995); Phys. Rev. E 52, 4134 (1995).

16 P. Gallo, M. Rovere, R. Pellarin, Europhys. Lett 57, 212 (2002).

17 H.R. Schober, J. Non-Cryst. Solids 307-310, 40 (2002). cond-mat/0112267

18 J.P. Boom and S. Yip, Molecular Hydrodynamics (Dover, New York, 1980).

19 F. Sciortino, P. Gallo, P. Tartaglia, S.-H. Chen Phys. Rev. E 54, 6331 (1996).

20 P. Gallo, R. Pellarin and M. Rovere, in preparation (2002).

21 P. Scheidler, W. Kob and K. Binder, Europhys. Lett. 52, 277 (2000); 59, 701 (2002). 